chloride. It seems clear that the incidence of such pains in patients receiving E.C.T. is lower than in reported series of patients not receiving this treatment and approximates to the incidence in patients who are kept in bed for 24 hours after receiving their anaesthetic. The incidence in relation to the number of anaesthetics given is much lower than that in any other series.

This finding appears to be a direct contradiction of the reports that the incidence of muscle pain is lowered by rest after the anaesthetic or by the reduction of the muscle activity consequent upon depolarization by the prior administration of nondepolarizing muscle relaxants. A direct relation between muscular pain and physical illness may be possible. If this were so, however, we should expect out-patients to be less physically ill than in-patients and therefore to have less pain. This has not been the case in those series where E.C.T. has not been given.

It may be possible to revive and modify Currie's (1953) original suggestion. He thought that the chest pain which his patients reported might be associated with overenthusiastic ventilation of the paralysed patient. The main difference between our patients and those in other series is the induction of a generalized fit, with muscle contractions which are visible despite the effect of the muscle relaxant. It may be possible to argue that the pains are related to the period of flaccidity which the patients undergo. This may have an effect related either to physical distortion of flaccid muscle or to some biochemical change related to this inactivity. For instance, metabolites may accumulate in inactive muscles which give rise to pain if the muscles are exercised immediately power returns. Such a mechanism may account for the differences between our series and the comparable out-patients series, and also for the previously observed differences between out-patients and in-patients.

\section{Summary and Conclusions}

The incidence of headaches and muscular pain arising after E.C.T. was investigated in 47 ambulant psychiatric patients who received a total of 259 treatments. Thirteen patients complained of muscular pain on a total of 22 occasions. Twenty-seven patients reported headaches on a total of 62 occasions. Headache was less common on the first treatment than on subsequent treatments.

The incidence of muscular pain in relation to the number of patients treated was significantly lower than in previous reports on out-patient series of patients who did not receive E.C.T. $(\mathbf{P}<0.001)$.

The incidence of muscular pain in relation to the number of anaesthetics given is lower than that in previously reported series of patients not receiving E.C.T. to a highly significant extent $(\mathrm{P}<0.0005)$.

We should like to thank Professor E. Stengel for arousing our interest in problems of pain in psychiatric patients, and our colleagues in the Department of Anaesthetics for their co-operation.

\section{REFERENCES}

Burtles, R., and Tunstall, M. E. (1961). Brit. F. Anaesth., 33, 24.

Churchill-Davidson, H. C. (1954). Brit. med. ¥., 1, 74.

Currie, T. T. (1953). Ibid., 1, 1032.

Engel, G. L. (1959). Amer. भ. Med., 26, 899.

Foster, C. A. (1960). Brit. med. 7., $2,24$.

Hegarty, P. (1956). Brit. 尹. Anaesth., 28, 209.

Leatherdale, R. A. L., Mayhew, R. A. J., and Hayton-Williams, D. S. (1959). Brit. med. F., 1, 904.

Maxwell, A. E. (1961). Analysing Qualitative Data. Methuen, London.

Morris, D. D. B., and Dunn, C. H. (1957). Brit. med. Ұ., 1, 383.

Prince-White, F.'(1957). Ibid., 1, 761.

Szasz, T. S. (1957). Pain and Pleasure. Basic Books, New York.

Tewfik, G. I. (1963). Brit. med. 7., 2, 251.

\title{
Adherence of Bath Oil to Keratin
}

\author{
JOHN M. KNOX,* M.D.; RYOHEI OGURA,* M.D., PH.D.
}

Self-emulsifying oils are being used to treat patients with dermatological diseases in which the skin is abnormally dry. Formulations of this type were commercially introduced after it was demonstrated that mixing recently developed surfaceactive agents with an oil gave the oil self-emulsifying properties (Knox et al., 1956, 1958). Although several of these formulations are available their use has not been extensively studied.

Taylor $(1961,1963)$ reported that mineral-oil products adhere better to the skin than do vegetable-oil formulations and that oilated oatmeal preparations leave little residual oil on the skin. In his two studies the patient's arm or body was immersed and the loss of oil from the emulsion was considered to be the amount of oil adhering to skin. The quantity of oil absorbed seemed directly proportional to the concentration of oil in the emulsion. It increased with higher bath-water temperatures and with longer incubation times, up to 20 minutes. The skin of patients with atopy, psoriasis, neurodermatitis, and ichthyosis absorbed approximately $70 \%$ more oil than did normal skin. Clinical studies attesting to the therapeutic effectiveness of these

* From the Department of Dermatology, Baylor University College of Medicine, Houston, Texas. agents have been published by James $(1959,1961)$, Spoor (1958), and Lubowe (1960).

This is a report of a series of experiments performed to confirm and further delineate factors which influence the adherence of bath oil to keratin, and to establish a laboratory technique for evaluating these factors. These studies used normal keratin, defatted keratin, psoriasis scales, and defatted psoriatic scales.

\section{Materials and Methods}

Stratum corneum and psoriatic scale were pulverized in a Willy laboratory mill to make particles that could pass through a 40-mesh screen. In some experiments test material was defatted with ether in a Soxhlet reflex distillation apparatus.

Four commercially available bath oils and a variety of formulations prepared by our pharmacists were selected for study. Bath-oil formulations were emulsified with distilled water in the desired concentrations and distilled water was used as the control. The quantitative procedure employed to determine the amount of oil absorbed by keratin was as follows. 
For each experiment $100 \mathrm{mg}$. of keratin was weighed in a small beaker and $5 \mathrm{ml}$. of the test emulsion added. This mixture was stirred with a glass rod and then allowed to stand for 10 minutes at room temperature. The study material was filtered with a Buchner funnel under reduced pressure from a vacuum pump. Residual keratin was washed with more emulsion $(1 \mathrm{ml}$.) of the same concentration (final volume $6 \mathrm{ml}$.). Then the washed keratin was dried in a vacuum desiccator and transferred to a centrifuge-tube to which $0.5 \mathrm{ml}$. of ether was added, stirred, and centrifuged for one minute. The supernatant solution was decanted into a small $10-\mathrm{ml}$. beaker of known weight. Ether extraction was repeated twice; then the ether was gently evaporated and the beaker weighed.

By subtracting the amount of oil normally present in the control sample from the amount of oil found in each test sample the remainder was considered to be the amount of oil absorbed. Normal keratin had a value of $15 \pm 0.55$ and psoriatic scale $116 \pm 2.13$. The amount of oil absorbed by keratin was calculated, and is presented as $\mathrm{mg}$./g. of keratin. All data were subjected to statistical analysis to determine if results were significant at the 0.05 level.

\section{Results}

Experiment 1: Adherence of Mineral Oil Versus Vegetable Oil.-Mineral oil, mineral oil plus $10 \%$ oleic acid, olive oil, and Wesson oil, each containing $10 \%$ of an alkylaryl polyether alcohol emulsifier, ${ }^{1}$ were emulsified with distilled water and compared. A $1 \%$ concentration was selected for comparative

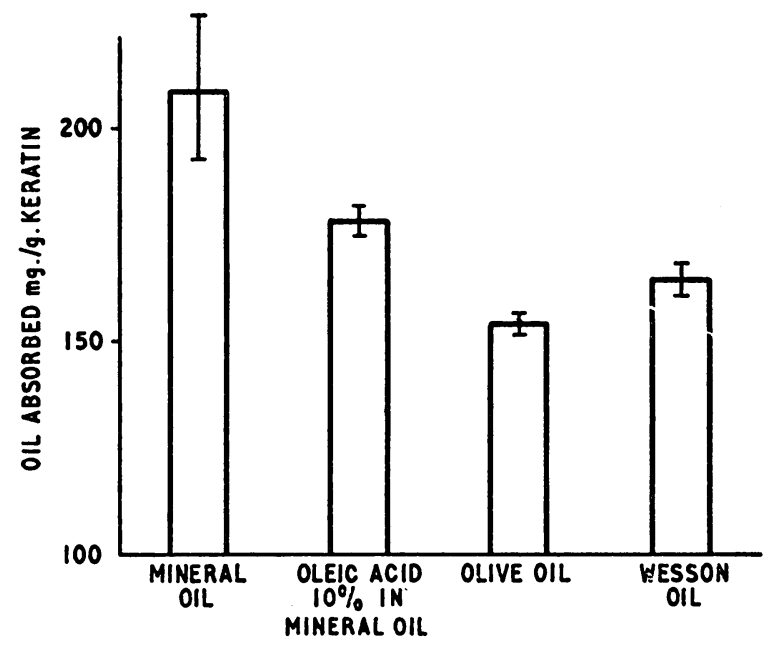

FIG. 1.-Mineral-oil and vegetable-oil formulations.

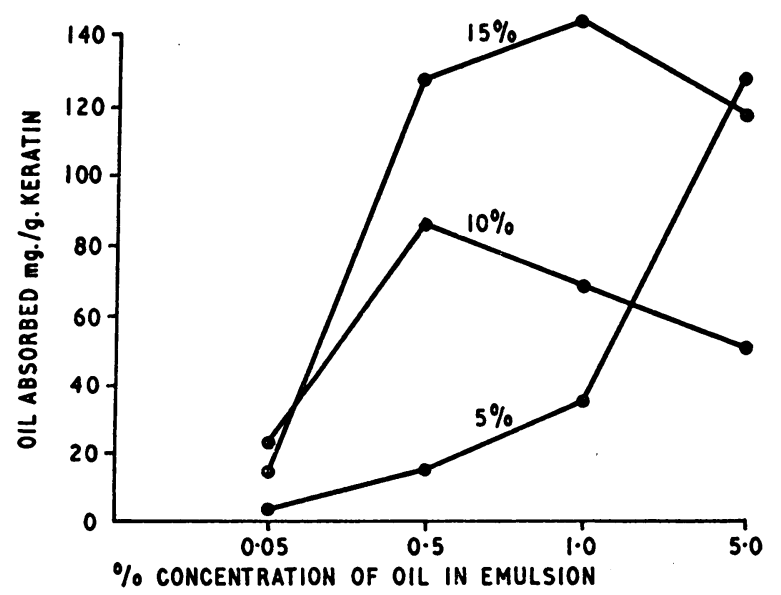

FIG. 2.-Variation in emulsifier concentration. $5 \%, 10 \%$, and $15 \%$ alkylaryl polyether alcohol. Ten minutes at room

'Triton X45, Rohm \& Haas Co.. Washington Square, Philadelphia, Penn. evaluation. Fig. 1 shows that mineral-oil formulations adhered better to keratin than did vegetable-oil preparations. Adding a coupling agent, oleic acid, to mineral oil improved the appearance of the emulsion but decreased its effectiveness.

Experiment 2 : Variation in Emulsifier Concentration.-Concentrations of 5,10 , and $15 \%$ of the emulsifier in mineral oil were studied by the above technique. Fig. 2 shows that the amount of oil absorbed was modified by the concentration of emulsifier. Adherence did not necessarily correlate with the physical excellence of the final emulsion.

Experiment 3: Normal and Pathological Keratin.-When normal and psoriatic-scale keratin were compared, the differences were striking in both defatted and non-defatted material. Psoriatic scale absorbed considerably more oil ${ }^{2}$ than normal keratin (Fig. 3) ; defatted material absorbed less oil than did non-defatted study samples.
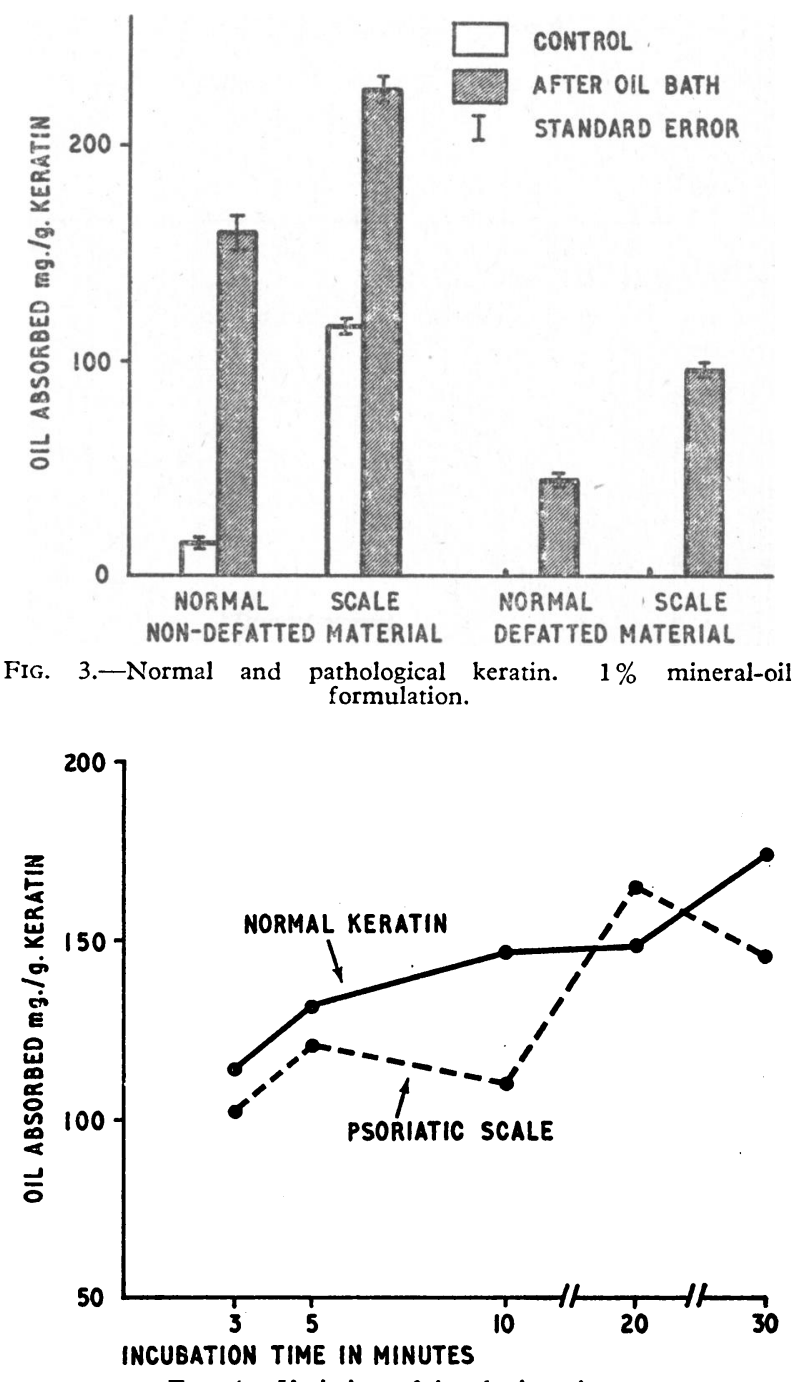

FIG. 4.-Variation of incubation time.

Experiment 4: Duration of Immersion.-A widely used mineral-oil formulation in a $1 \%$ concentration was employed for this experiment. Immersion periods varied from 3 to 30 minutes. Fig. 4 indicates that adherence to keratin increases with the length of exposure.

Experiment 5 : Effect of Temperature.-In this experiment the same formulation and concentration was used as in Experiment 3, but the bath-water temperatures were 75,90 , and $105^{\circ}$ F. $\left(23.9,32.2\right.$, and $40.6^{\circ}$ C.). Oil-absorption increased

$21 \%$ proprietary mineral-oil formulation-Alpha-Keri Water Dispersible Antipruritic Oil, Westwood Pharmaceuticals, Buffalo, N.Y. 
with the temperature of the bath (Fig. 5). The increase in adherence was spectacular with psoriatic scale.

Experiment 6 : Comparison of Commercial Oil-bath Formulations.-Figs. 6 and 7 demonstrate that the four bath-oil products had significantly different absorption characteristics. With this analytical technique the two mineral-oil formulations (A and $B$ ) were superior to a vegetable-oil (C) and a foaming-type formulation (D). Even the two mineral-oil formulations varied significantly in their adherence to keratin.

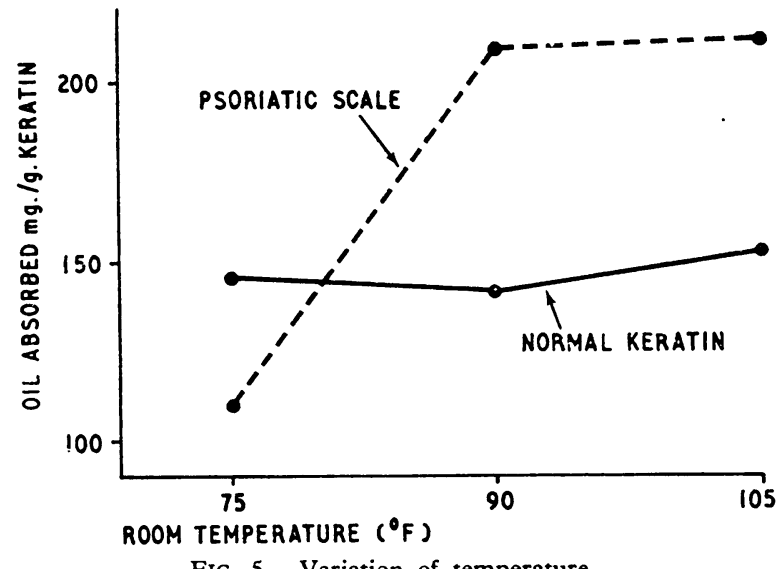

FIG. 5.-Variation of temperature.

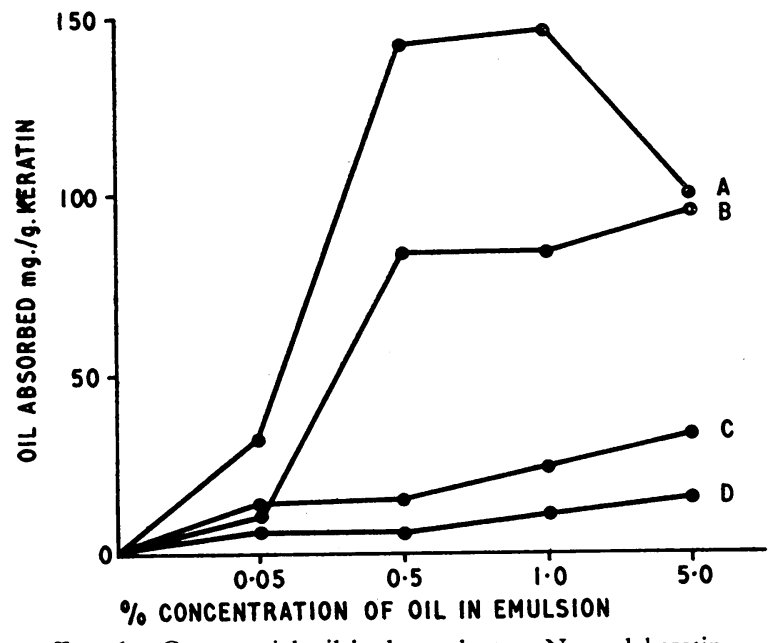

FIG. 6.-Commercial oil-bath products. Normal keratin.

\section{Discussion}

Emulsion concentrations of less than $0.05 \%$ were not used because preliminary investigations revealed that reproducible results were difficult to obtain when lower concentrations were employed. In treating patients the concentrations normally are about $0.02 \%$; therefore our in vitro laboratory study may not duplicate a clinical situation. Although this technique may squal or be even more accurate and reliable than the method used by Taylor, a more sensitive analytical laboratory procedure possibly is needed. However, the techniques produced somewhat similar results, so perhaps each procedure is reliable.

Successful treatment probably does not depend entirely on quantitative oil-adherence to keratin. Size of droplet and the physical or chemical properties of the oil may be important.

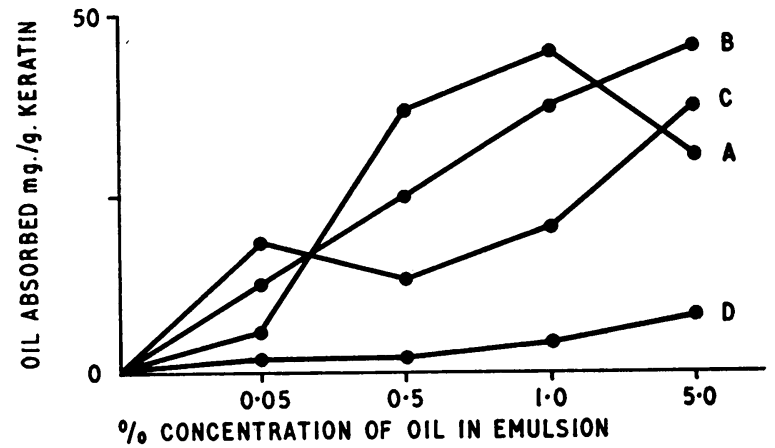

FIG. 7.-Commercial oil-bath products. Defatted keratin.

Defatted stratum corneum and scale were included because oil baths are often advocated for pathologically dry and scaling skin diseases. However, it is recognized that ether extraction defatting does not duplicate the clinical situation.

The results of these experiments indicate that considerable care is needed in arriving at the optional formula for a selfemulsifying bath oil because ability to disperse in water is not the only criterion for the adherence of oil to keratin.

\section{Summary}

The relative lubricating effectiveness of commercial oil-bath formulations has received little attention. In these experiments keratin was pulverized, then incubated in several different bathoil formulations. Adherence to keratin was determined by an ether-extraction technique.

Mineral-oil preparations adhered to keratin better than did vegetable-oil preparations. Oil-absorption increased with the duration of immersion and temperature of the bath. Psoriatic scale absorbed considerably more oil than did normal keratin, whereas defatted material absorbed less than did non-defatted material.

The many factors which determine adherence to keratin must be carefully considered in the search for an optimal formula for a self-emulsifying bath oil.

\section{REFERENCES}

Knox, J. M., Everett, M. A., and Curtis, A. C. (1956). Arch. Derm. 74, 699.

- (1958). Ibid., 78, 642.

James, A. P. R. (1959). Sthwest. Med., 40, 384

(1961). F. Amer. Geriat. Soc., 9, 367.

Lubowe, I. I. (1960). West. Med., p. 45, Dec.

Spoor, H. J. (1958). N.Y. St. F. Med., 58, 3292

Taylor, E. A. (1901). F. invest. Derm., 37, 69.

(1963). Arch. Derm., 87, 369. 